\title{
UPPER SEMICONTINUITY OF THE ATTRACTOR FOR LATTICE DYNAMICAL SYSTEMS OF PARTLY DISSIPATIVE REACTION-DIFFUSION SYSTEMS
}

\author{
AHMED Y. ABDALLAH \\ Received 12 July 2004 and in revised form 7 December 2004
}

We investigate the existence of a global attractor and its upper semicontinuity for the infinite-dimensional lattice dynamical system of a partly dissipative reaction-diffusion system in the Hilbert space $l^{2} \times l^{2}$. Such a system is similar to the discretized FitzHughNagumo system in neurobiology, which is an adequate justification for its study.

\section{Introduction}

Lattice dynamical systems arise in various application fields, for instance, in chemical reaction theory, material science, biology, laser systems, image processing and pattern recognition, and electrical engineering (cf. $[6,7,13])$. In each field, they have their own forms, but in some other cases, they appear as spatial discretizations of partial differential equations (PDEs). Recently, many authors studied various properties of the solutions for several lattice dynamical systems. For instance, the chaotic properties have been investigated in $[1,7,8,10,11,17]$, and the travelling solutions have been carefully studied in $[2,3,7,8,9,22]$.

From [18], we know that it is difficult to estimate the attractor of the solution semiflow generated by the initial value problem of dissipative PDEs on unbounded domains because, in general, it is infinite dimensional. Therefore, it is significant to study the lattice dynamical systems corresponding to the initial value problem of PDEs on unbounded domains because of the importance of such systems and they can be regarded as an approximation to the corresponding continuous PDEs if they arise as spatial discretizations of PDEs.

The main idea of this work is originated from $[4,16]$. In $[4,19,20]$, the researchers proved the existence of global attractors for different lattice dynamical systems and they investigated the finite-dimensional approximations of these global attractors. In fact, Bates et al. [4] studied first-order lattice dynamical systems, and Zhou [20] gave a generalization of the result given by [4]. Zhou [19] studied a second-order lattice dynamical system and investigated the upper semicontinuity of the global attractor. 
For a positive integer $r$, consider the Hilbert space

$$
l^{2}=\left\{u=\left(u_{i}\right)_{i \in \mathbb{Z}^{r}}: i=\left(i_{1}, i_{2}, \ldots, i_{r}\right) \in \mathbb{Z}^{r}, u_{i} \in \mathbb{R}, \sum_{i \in \mathbb{Z}^{r}} u_{i}^{2}<\infty\right\}
$$

whose inner product and norm are given by, for all $u=\left(u_{i}\right)_{i \in \mathbb{Z}^{r}}, v=\left(v_{i}\right)_{i \in \mathbb{Z}^{r}} \in l^{2}$,

$$
\langle u, v\rangle=\sum_{i \in \mathbb{Z}^{r}} u_{i} v_{i}, \quad\|u\|=\langle u, u\rangle^{1 / 2}=\left(\sum_{i \in \mathbb{Z}^{r}} u_{i}^{2}\right)^{1 / 2} .
$$

We will study the following lattice dynamical system of a partly dissipative reactiondiffusion system:

$$
\begin{gathered}
\dot{u}_{i}+v(A u)_{i}+f_{1}\left(u_{i}\right)+g_{1}\left(u_{i}\right)+\alpha h_{1}\left(u_{i}, v_{i}\right)=q_{1, i}, \\
\dot{v}_{i}+f_{2}\left(v_{i}\right)+g_{2}\left(v_{i}\right)-\beta h_{2}\left(u_{i}, v_{i}\right)=q_{2, i},
\end{gathered}
$$

$i=\left(i_{1}, i_{2}, \ldots, i_{r}\right) \in \mathbb{Z}^{r}, t>0$, with the initial conditions

$$
u_{i}(0)=u_{i, 0}, \quad v_{i}(0)=v_{i, 0},
$$

where $\nu$ is a positive constant, $\alpha$ and $\beta$ are real constants such that

$$
\alpha \beta>0
$$

the operator $A: l^{2} \rightarrow l^{2}$ is defined by, for all $u=\left(u_{i}\right)_{i \in \mathbb{Z}^{r}} \in l^{2}, i=\left(i_{1}, i_{2}, \ldots, i_{r}\right)$,

$$
\begin{aligned}
(A u)_{i}= & 2 r u_{\left(i_{1}, i_{2}, \ldots, i_{r}\right)}-u_{\left(i_{1}-1, i_{2}, \ldots, i_{r}\right)}-u_{\left(i_{1}, i_{2}-1, \ldots, i_{r}\right)}-\cdots-u_{\left(i_{1}, i_{2}, \ldots, i_{r}-1\right)} \\
& -u_{\left(i_{1}+1, i_{2}, \ldots, i_{r}\right)}-u_{\left(i_{1}, i_{2}+1, \ldots, i_{r}\right)}-\cdots-u_{\left(i_{1}, i_{2}, \ldots, i_{r}+1\right)},
\end{aligned}
$$

and for $j=1,2, s, s_{1}, s_{2} \in \mathbb{R}$,

$$
\begin{gathered}
q_{j}=\left(q_{j, i}\right)_{i \in \mathbb{Z}^{r}} \in l^{2}, \\
f_{j}(s), g_{j}(s) \in C^{1}(\mathbb{R}, \mathbb{R}), \quad f_{j}(0)=g_{j}(0)=0, \\
h_{j}\left(s_{1}, s_{2}\right) \in C^{1}\left(\mathbb{R}^{2}, \mathbb{R}\right), \quad h_{j}(0,0)=0, \\
\varepsilon_{j} \leq f_{j}^{\prime}(s), \\
g_{j}(s) s \geq 0, \\
h_{1}\left(s_{1}, s_{2}\right) s_{1}=h_{2}\left(s_{1}, s_{2}\right) s_{2},
\end{gathered}
$$

where $\varepsilon_{j}$ is a positive constant. 
For example, one can consider $f_{j}(s)=\delta_{j} s$, where $\delta_{j}$ is a positive constant, $g_{j}(s)=$ $\sum_{i=0}^{n_{j}} \gamma_{i j} s^{2 i+1}$, where $n_{j}$ is a nonnegative integer and $\gamma_{i j}$ is a nonnegative constant for each $i=0,1, \ldots, n_{j}, h_{1}\left(s_{1}, s_{2}\right)=s_{1}^{m_{1}-1} s_{2}^{m_{2}}$, and $h_{2}\left(s_{1}, s_{2}\right)=s_{1}^{m_{1}} s_{2}^{m_{2}-1}$, where $m_{1}, m_{2} \geq 2$ are constants. In fact, for $f_{1}(s)=\lambda s, f_{2}(s)=\delta s, g_{2}(s)=0, h_{1}\left(s_{1}, s_{2}\right)=s_{2}$, and $h_{2}\left(s_{1}, s_{2}\right)=s_{1}$, where $\lambda$ and $\delta$ are positive constants, the system given by (1.3) and (1.4) can be regarded as a spatial discretization of the following partly dissipative reaction-diffusion system with continuous spatial variable $x \in \mathbb{R}^{r}$ and $t \in \mathbb{R}^{+}$:

$$
u_{t}-v \Delta u+\lambda u+g_{1}(u)+\alpha v=q_{1}, \quad v_{t}+\delta v-\beta u=q_{2} .
$$

The system (1.13) describes the signal transmission across axons and is a model of FitzHugh-Nagumo equations in neurobiology, (cf. $[5,12,15]$ and the references therein). The existence of global attractors of the system given by (1.13) has been proved in a bounded domain (cf. [14]) and in $\mathbb{R}^{r}$ (cf. [16]).

\section{Preliminaries}

We can write the operator $A$ in the following form:

$$
A=A_{1}+A_{2}+\cdots+A_{r}
$$

such that for $j=1,2, \ldots, r$, and $u=\left(u_{i}\right)_{i \in \mathbb{Z}^{r}} \in l^{2}$,

$$
\left(A_{j} u\right)_{i}=2 u_{\left(i_{1}, i_{2}, \ldots, i_{r}\right)}-u_{\left(i_{1}, i_{2}, \ldots, i_{j-1}, i_{j}-1, i_{j+1}, \ldots, i_{r}\right)}-u_{\left(i_{1}, i_{2}, \ldots, i_{j-1}, i_{j}+1, i_{j+1}, \ldots, i_{r}\right)} .
$$

For $j=1,2, \ldots, r$, define the operators $B_{j}, B_{j}^{*}: l^{2} \rightarrow l^{2}$ as follows: for $u=\left(u_{i}\right)_{i \in \mathbb{Z}^{r}} \in l^{2}$,

$$
\begin{aligned}
\left(B_{j} u\right)_{i} & =u_{\left(i_{1}, i_{2}, \ldots, i_{j-1}, i_{j}+1, i_{j+1}, \ldots, i_{r}\right)}-u_{\left(i_{1}, i_{2}, \ldots, i_{r}\right)}, \\
\left(B_{j}^{*} u\right)_{i} & =u_{\left(i_{1}, i_{2}, \ldots, i_{j-1}, i_{j}-1, i_{j+1}, \ldots, i_{r}\right)}-u_{\left(i_{1}, i_{2}, \ldots, i_{r}\right) .}
\end{aligned}
$$

Then, it follows that for $j=1,2, \ldots, r$,

$$
A_{j}=B_{j}^{*} B_{j}=B_{j} B_{j}^{*}
$$

there exists a constant $C_{0}=C_{0}(r)$ such that

$$
\begin{gathered}
\|A u\|^{2} \leq C_{0}\|u\|^{2}, \quad\left\|B_{j} u\right\|^{2}=\left\|B_{j}^{*} u\right\|^{2} \leq 4\|u\|^{2}, \quad \forall u \in l^{2}, \\
\left\langle B_{j} u, v\right\rangle=\left\langle u, B_{j}^{*} v\right\rangle, \quad \forall u, v \in l^{2} .
\end{gathered}
$$

It is clear that $A, A_{j}, B_{j}$, and $B_{j}^{*}, j=1,2, \ldots, r$, are bounded linear operators from $l^{2}$ into $l^{2}$.

We can represent the initial value problem, (1.3) and (1.4), in the following form:

$$
\begin{gathered}
\dot{u}+v A u+f_{1}(u)+g_{1}(u)+\alpha h_{1}(u, v)=q_{1}, \\
\dot{v}+f_{2}(v)+g_{2}(v)-\beta h_{2}(u, v)=q_{2}, \\
u(0)=\left(u_{i, 0}\right)_{i \in \mathbb{Z}^{r}}, \quad v(0)=\left(v_{i, 0}\right)_{i \in \mathbb{Z}^{r}},
\end{gathered}
$$


where $u=\left(u_{i}\right)_{i \in \mathbb{Z}^{r}}, v=\left(v_{i}\right)_{i \in \mathbb{Z}^{r}}, A u=\left(A u_{i}\right)_{i \in \mathbb{Z}^{r}}$, and for $j=1,2, f_{j}(u)=\left(f_{j}\left(u_{i}\right)\right)_{i \in \mathbb{Z}^{r}}$, $g_{j}(u)=\left(g_{j}\left(u_{i}\right)\right)_{i \in \mathbb{Z}^{r}}, h_{j}(u, v)=\left(h_{j}\left(u_{i}, v_{i}\right)\right)_{i \in \mathbb{Z}^{r}}, q_{j}=\left(q_{j, i}\right)_{i \in \mathbb{Z}^{r}}$.

Consider the Hilbert space $E:=l^{2} \times l^{2}$, endowed with the inner product and norm as follows: for $\varphi_{j}=\left(u^{(j)}, v^{(j)}\right)^{T}=\left(\left(u_{i}^{(j)}\right),\left(v_{i}^{(j)}\right)\right)_{i \in \mathbb{Z}^{r}}^{T} \in E, j=1,2$,

$$
\begin{gathered}
\left\langle\varphi_{1}, \varphi_{2}\right\rangle_{E}=\left\langle u^{(1)}, u^{(2)}\right\rangle+\left\langle v^{(1)}, v^{(2)}\right\rangle, \\
\|\varphi\|_{E}=\langle\varphi, \varphi\rangle_{E}^{1 / 2}, \quad \forall \varphi \in E .
\end{gathered}
$$

Now, the system given by (2.7) is equivalent to the following initial value problem in the Hilbert space $E:=l^{2} \times l^{2}$ :

$$
\dot{\varphi}+C(\varphi)=F(\varphi), \quad \varphi(0)=\left(u_{0}, v_{0}\right)^{T},
$$

where $\varphi=(u, v)^{T}, C(\varphi)=(\nu A u, 0)^{T}, F(\varphi)=\left(G_{1}(\varphi), G_{2}(\varphi)\right)^{T}$,

$$
\begin{aligned}
& G_{1}(\varphi)=q_{1}-f_{1}(u)-g_{1}(u)-\alpha h_{1}(u, v), \\
& G_{2}(\varphi)=q_{2}-f_{2}(v)-g_{2}(v)+\beta h_{2}(u, v) .
\end{aligned}
$$

For a given function $f\left(s_{1}, s_{2}\right) \in C^{1}\left(\mathbb{R}^{2}, \mathbb{R}\right)$, let $D_{1} f(a, b)$ represent the partial derivative of $f$ with respect to the first independent variable, $s_{1}$, at $\left(s_{1}, s_{2}\right)=(a, b)$, and let $D_{2} f(a, b)$ represent the partial derivative of $f$ with respect to the second independent variable, $s_{2}$, at $\left(s_{1}, s_{2}\right)=(a, b)$. From (1.9) and the mean value theorem, it follows that given $u=\left(u_{i}\right)_{i \in \mathbb{Z}^{r}}$, $v=\left(v_{i}\right)_{i \in \mathbb{Z}^{r}} \in l^{2}$, there exist $\xi_{1 i}, \xi_{2 i} \in(0,1)$, for each $i \in \mathbb{Z}^{r}$, such that

$$
\begin{aligned}
\left\|h_{1}(u, v)\right\|^{2}= & \sum_{i \in \mathbb{Z}^{r}}\left(h_{1}\left(u_{i}, v_{i}\right)\right)^{2}=\sum_{i \in \mathbb{Z}^{r}}\left(D_{1} h_{1}\left(\xi_{1 i} u_{i}, v_{i}\right) u_{i}+D_{2} h_{1}\left(0, \xi_{2 i} v_{i}\right) v_{i}\right)^{2} \\
\leq & 2\left(\max _{|a| \leq\|u\||b| \leq\|v\|} \max _{1}\left(D_{1} h_{1}(a, b)\right)^{2}\right)\|u\|^{2} \\
& +2\left(\max _{|b| \leq\|v\|}\left(D_{2} h_{1}(0, b)\right)^{2}\right)\|v\|^{2}
\end{aligned}
$$

Thus, for $u, v \in l^{2}$, we have $h_{1}(u, v) \in l^{2}$. Similarly one can show that for $u, v \in l^{2}, h_{2}(u, v)$ $\in l^{2}$. By using (1.8) and the mean value theorem, it is easy to show that $f_{1}, f_{2}, g_{1}$, and $g_{2}$ map $l^{2}$ into $l^{2}$. From the above discussion, it is obvious that $F$ maps $E$ into $E$.

\section{The existence of an absorbing set}

First we will prove that there exists a unique local solution of the system given by (2.9) in E. Suppose that (1.7), (1.8), and (1.9) are satisfied. Let $G$ be a bounded set in $E$, and 
$\varphi_{j}=\left(u^{(j)}, v^{(j)}\right)=\left(\left(u_{i}^{(j)}\right),\left(v_{i}^{(j)}\right)\right)_{i \in \mathbb{Z}^{r}} \in G, j=1,2$, then

$$
\begin{aligned}
\| F\left(\varphi_{1}\right) & -F\left(\varphi_{2}\right) \|_{E}^{2} \\
= & \left\|G_{1}\left(\varphi_{1}\right)-G_{1}\left(\varphi_{2}\right)\right\|^{2}+\left\|G_{2}\left(\varphi_{1}\right)-G_{2}\left(\varphi_{2}\right)\right\|^{2} \\
\leq & 2\left\|f_{1}\left(u^{(1)}\right)-f_{1}\left(u^{(2)}\right)\right\|^{2}+4\left\|g_{1}\left(u^{(1)}\right)-g_{1}\left(u^{(2)}\right)\right\|^{2} \\
& +4 \alpha^{2}\left\|h_{1}\left(u^{(1)}, v^{(1)}\right)-h_{1}\left(u^{(2)}, v^{(2)}\right)\right\|^{2}+2\left\|f_{2}\left(v^{(1)}\right)-f_{2}\left(v^{(2)}\right)\right\|^{2} \\
& +4\left\|g_{2}\left(v^{(1)}\right)-g_{2}\left(v^{(2)}\right)\right\|^{2}+4 \beta^{2}\left\|h_{2}\left(u^{(1)}, v^{(1)}\right)-h_{2}\left(u^{(2)}, v^{(2)}\right)\right\|^{2} .
\end{aligned}
$$

Using (1.9) and the mean value theorem, it follows that there exist $\xi_{3 i}, \xi_{4 i} \in(0,1)$, for each $i \in \mathbb{Z}^{r}$, and $L_{1}=L_{1}(G)$ such that

$$
\begin{aligned}
&\left\|h_{1}\left(u^{(1)}, v^{(1)}\right)-h_{1}\left(u^{(2)}, v^{(2)}\right)\right\|^{2} \\
&=\sum_{i \in \mathbb{Z}^{r}}\left(h_{1}\left(u_{i}^{(1)}, v_{i}^{(1)}\right)-h_{1}\left(u_{i}^{(2)}, v_{i}^{(2)}\right)\right)^{2} \\
&=\sum_{i \in \mathbb{Z}^{r}}\left(\begin{array}{c}
D_{1} h_{1}\left(u_{i}^{(1)}+\xi_{3 i}\left(u_{i}^{(2)}-u_{i}^{(1)}\right), v_{i}^{(1)}\right)\left(u_{i}^{(1)}-u_{i}^{(2)}\right) \\
+D_{2} h_{1}\left(u_{i}^{(2)}, v_{i}^{(1)}+\xi_{4 i}\left(v_{i}^{(2)}-v_{i}^{(1)}\right)\right)\left(v_{i}^{(1)}-v_{i}^{(2)}\right)
\end{array}\right)^{2} \\
& \leq 2\left(\max _{|a| \leq\left\|u^{(1)}\right\|+\left\|u^{(2)}\right\|,|b| \leq\left\|v^{(1)}\right\|}\left(D_{1} h_{1}(a, b)\right)^{2}\right)\left\|u^{(1)}-u^{(2)}\right\|^{2} \\
& \quad+2\left(\max _{|a| \leq\left\|u^{(2)}\right\|,|b| \leq\left\|v^{(1)}\right\|+\left\|v^{(2)}\right\|}\left(D_{2} h_{1}(a, b)\right)^{2}\right)\left\|v^{(1)}-v^{(2)}\right\|^{2} \\
& \leq L_{1}\left(\left\|u^{(1)}-u^{(2)}\right\|^{2}+\left\|v^{(1)}-v^{(2)}\right\|^{2}\right) .
\end{aligned}
$$

Thus

$$
\left\|h_{1}\left(u^{(1)}, v^{(1)}\right)-h_{1}\left(u^{(2)}, v^{(2)}\right)\right\|^{2} \leq L_{1}\left\|\varphi_{1}-\varphi_{2}\right\|^{2} .
$$

We can obtain similar results, as (3.3), for $f_{1}, f_{2}, g_{1}, g_{2}$, and $h_{2}$ by using (1.8), (1.9), and the mean value theorem. In such a case, using (3.1), there exists $L_{2}=L_{2}(G)$ such that

$$
\left\|F\left(\varphi_{1}\right)-F\left(\varphi_{2}\right)\right\|_{E}^{2} \leq L_{2}\left\|\varphi_{1}-\varphi_{2}\right\|^{2}
$$

Thus $F$ is locally Lipschitz from $E$ into $E$. In such a case from the standard theory of ordinary differential equations, we get the following lemma.

Lemma 3.1. If (1.7), (1.8), and (1.9) are satisfied, then for any initial data $\varphi(0)=\left(u_{0}\right.$, $\left.v_{0}\right)^{T} \in E$, there exists a unique local solution $\varphi(t)=(u(t), v(t))^{T}$ of (2.9) such that $\varphi \in$ $C^{1}([0, T), E)$ for some $T>0$. If $T<\infty$, then $\lim _{t \rightarrow T^{-}}\|\varphi\|_{E}^{2}=\infty$.

Assume that (1.5), (1.7), (1.8), (1.9), (1.10), (1.11), and (1.12) are satisfied. Now we are ready to prove that the solution of the system given by (2.9) exists globally and there exists an absorbing set. 
Let $\varphi=(u, v)^{T} \in E$ be a solution of (2.9). If we consider the inner product of (2.9) with $\varphi$ in $E$, taking into account (2.4) and (2.6), we obtain

$$
\begin{gathered}
\frac{1}{2} \frac{d}{d t}\|u\|^{2}+v \sum_{j=1}^{r}\left\|B_{j} u\right\|^{2}+\left\langle f_{1}(u), u\right\rangle+\left\langle g_{1}(u), u\right\rangle+\alpha\left\langle h_{1}(u, v), u\right\rangle=\left\langle q_{1}, u\right\rangle \\
\frac{1}{2} \frac{d}{d t}\|v\|^{2}+\left\langle f_{2}(v), v\right\rangle+\left\langle g_{2}(v), v\right\rangle-\beta\left\langle h_{2}(u, v), v\right\rangle=\left\langle q_{2}, v\right\rangle
\end{gathered}
$$

If we multiply (3.5) by $|\beta|$, (3.6) by $|\alpha|$, and we sum up the results, taking into account (1.5) and (1.12), we find that

$$
\begin{aligned}
\frac{|\beta|}{2} \frac{d}{d t}\|u\|^{2}+\frac{|\alpha|}{2} \frac{d}{d t} \| & v\left\|^{2}+|\beta| v \sum_{j=1}^{r}\right\| B_{j} u \|^{2}+|\beta|\left\langle f_{1}(u), u\right\rangle+|\alpha|\left\langle f_{2}(v), v\right\rangle \\
& +|\beta|\left\langle g_{1}(u), u\right\rangle+|\alpha|\left\langle g_{2}(v), v\right\rangle=|\beta|\left\langle q_{1}, u\right\rangle+|\alpha|\left\langle q_{2}, v\right\rangle .
\end{aligned}
$$

By using (1.8), (1.10), and the mean value theorem, for each $i \in \mathbb{Z}^{r}$, there exists a constant $\xi_{5 i} \in(0,1)$ such that

$$
\left\langle f_{1}(u), u\right\rangle=\sum_{i \in \mathbb{Z}^{r}}\left(f_{1}\left(u_{i}\right) u_{i}\right)=\sum_{i \in \mathbb{Z}^{r}}\left(f_{1}^{\prime}\left(\xi_{5 i} u_{i}\right) u_{i}^{2}\right) \geq \varepsilon_{1}\|u\|^{2}
$$

Thus we have

$$
\left\langle f_{1}(u), u\right\rangle \geq \varepsilon_{1}\|u\|^{2}, \quad\left\langle f_{2}(v), v\right\rangle \geq \varepsilon_{2}\|v\|^{2} .
$$

From (1.11), we obtain

$$
\left\langle g_{1}(u), u\right\rangle=\sum_{i \in \mathbb{Z}^{r}}\left(g_{1}\left(u_{i}\right) u_{i}\right) \geq 0, \quad\left\langle g_{2}(v), v\right\rangle \geq 0 .
$$

Now if we substitute (3.9) and (3.10) into (3.7), we find that

$$
\begin{aligned}
& \frac{|\beta|}{2} \frac{d}{d t}\|u\|^{2}+\frac{|\alpha|}{2} \frac{d}{d t}\|v\|^{2}+\varepsilon_{1}|\beta|\|u\|^{2}+\varepsilon_{2}|\alpha|\|v\|^{2} \\
& \quad \leq|\beta|\left\langle q_{1}, u\right\rangle+|\alpha|\left\langle q_{2}, v\right\rangle \leq \frac{|\beta|}{2 \varepsilon_{1}}\left\|q_{1}\right\|^{2}+\frac{\varepsilon_{1}|\beta|}{2}\|u\|^{2}+\frac{|\alpha|}{2 \varepsilon_{2}}\left\|q_{2}\right\|^{2}+\frac{\varepsilon_{2}|\alpha|}{2}\|v\|^{2} .
\end{aligned}
$$

Thus if we choose

$$
\sigma=\min \left\{|\beta|,|\alpha|, \varepsilon_{1}|\beta|, \varepsilon_{2}|\alpha|\right\}
$$


then it follows that

$$
\frac{d}{d t}\|\varphi\|_{E}^{2}+\|\varphi\|_{E}^{2} \leq\left(\frac{\beta}{\sigma}\left\|q_{1}\right\|\right)^{2}+\left(\frac{\alpha}{\sigma}\left\|q_{2}\right\|\right)^{2} .
$$

From the Gronwall lemma, we obtain

$$
\begin{gathered}
\|\varphi\|_{E}^{2} \leq e^{-t}\left\|\varphi_{0}\right\|_{E}^{2}+\frac{1-e^{-t}}{\sigma^{2}}\left(\left(\beta\left\|q_{1}\right\|\right)^{2}+\left(\alpha\left\|q_{2}\right\|\right)^{2}\right), \\
\lim _{t \rightarrow \infty}\|\varphi\|_{E}^{2} \leq\left(\frac{\beta}{\sigma}\left\|q_{1}\right\|\right)^{2}+\left(\frac{\alpha}{\sigma}\left\|q_{2}\right\|\right)^{2}
\end{gathered}
$$

Inequality (3.15) implies that the solution semigroup $\{S(t)\}_{t \geq 0}$ of (2.9) exists globally and possesses a bounded absorbing set in $E$. In such a case, the maps

$$
S(t): \varphi(0) \in E \longrightarrow S(t) \varphi(0)=\varphi(t) \in E, \quad t \geq 0,
$$

generate a continuous semigroup $\{S(t)\}_{t \geq 0}$ on $E$. Now from Lemma 3.1 and (3.15), we are ready to present the following lemma.

Lemma 3.2. If (1.5), (1.7), (1.8), (1.9), (1.10), (1.11), and (1.12) are satisfied, then for any initial data in $E$, the solution $\varphi(t)$ of (2.9) exists globally for all $t \geq 0$. That is, $\varphi \in$ $C^{1}([0, \infty), E)$. Moreover, there exists a bounded ball $O=O_{E}\left(0, r_{0}\right)$ in $E$, centered at 0 with radius $r_{0}$, such that for every bounded set $G$ of $E$, there exists $T(G) \geq 0$ such that

$$
S(t) G \subset O, \quad \forall t \geq T(G)
$$

where $r_{0}^{2}>\left((\beta / \sigma)\left\|q_{1}\right\|\right)^{2}+\left((\alpha / \sigma)\left\|q_{2}\right\|\right)^{2}$. Therefore, there exists a constant $T_{0} \geq 0$ depending on $O$ such that

$$
S(t) O \subset O, \quad \forall t \geq T_{0}
$$

\section{The existence of the global attractor}

To prove the existence of the global attractor for the solution semigroup $\{S(t)\}_{t \geq 0}$ of (2.9), we need to prove the asymptotic compactness of $\{S(t)\}_{t \geq 0}$. Along the lines of [4], the key idea of showing the asymptotic compactness for such a lattice system is to establish uniform estimates on "Tail Ends" of solutions.

LEMmA 4.1. If (1.5), (1.7), (1.8), (1.9), (1.10), (1.11), and (1.12) are satisfied and $\varphi(0)=$ $\left(u_{0}, v_{0}\right) \in O$, where $O$ is the bounded absorbing ball given by Lemma 3.2, then for any $\eta>0$, there exist positive constants $T(\eta)$ and $K(\eta)$ such that the solution $\varphi(t)=(u(t), v(t))^{T}=$ $\left(\varphi_{i}(t)\right)_{i \in \mathbb{Z}^{r}}=\left(\left(u_{i}(t)\right),\left(v_{i}(t)\right)\right)_{i \in \mathbb{Z}^{r}}^{T} \in E$ of (2.9) satisfies

$$
\sum_{\|i\|_{m} \geq K(\eta)}\left\|\varphi_{i}(t)\right\|_{E}^{2}=\sum_{\|i\|_{m} \geq K(\eta)}\left(u_{i}^{2}(t)+v_{i}^{2}(t)\right) \leq \eta
$$

for all $t \geq T(\eta)$, where $\|i\|_{m}=\max _{1 \leq j \leq r}\left|i_{j}\right|$ for $i=\left(i_{1}, i_{2}, \ldots, i_{r}\right) \in \mathbb{Z}^{r}$. 
Proof. Consider a smooth increasing function $\theta \in C^{1}\left(\mathbb{R}^{+}, \mathbb{R}\right)$ such that

$$
\begin{aligned}
\theta(s)=0, & 0 \leq s<1, \\
0 \leq \theta(s) \leq 1, & 1 \leq s<2, \\
\theta(s)=1, & s \geq 2,
\end{aligned}
$$

and there exists a constant $M_{0}$ such that $\theta^{\prime}(s) \leq M_{0}$ for all $s \in \mathbb{R}^{+}$.

Let $L$ be an arbitrary positive integer. Set $w_{i}=\theta\left(\|i\|_{m} / L\right) u_{i}, z_{i}=\theta\left(\|i\|_{m} / L\right) v_{i}, w=$ $\left(w_{i}\right)_{i \in \mathbb{Z}^{r}}, z=\left(z_{i}\right)_{i \in \mathbb{Z}^{r}}$, and $\psi=(w, z)^{T}$. Following [4], we take the inner product of (2.9) with $\psi$ in $E$, then it follows that

$$
\begin{gathered}
\sum_{i \in \mathbb{Z}^{r}} \theta\left(\frac{\|i\|_{m}}{L}\right)\left(\frac{1}{2} \frac{d}{d t} u_{i}^{2}+f_{1}\left(u_{i}\right) u_{i}+g_{1}\left(u_{i}\right) u_{i}+\alpha h_{1}\left(u_{i}, v_{i}\right) u_{i}\right) \\
+v \sum_{i \in \mathbb{Z}^{r}} \sum_{j=1}^{r}\left(B_{j} u\right)_{i}\left(B_{j} w\right)_{i}=\sum_{i \in \mathbb{Z}^{r}} \theta\left(\frac{\|i\|_{m}}{L}\right) q_{1, i} u_{i}, \\
\sum_{i \in \mathbb{Z}^{r}} \theta\left(\frac{\|i\|_{m}}{L}\right)\left(\frac{1}{2} \frac{d}{d t} v_{i}^{2}+f_{2}\left(v_{i}\right) v_{i}+g_{2}\left(v_{i}\right) v_{i}-\beta h_{2}\left(u_{i}, v_{i}\right) v_{i}\right)=\sum_{i \in \mathbb{Z}^{r}} \theta\left(\frac{\|i\|_{m}}{L}\right) q_{2, i} v_{i} .
\end{gathered}
$$

If we multiply (4.3) by $|\beta|$, (4.4) by $|\alpha|$, and we sum up the results, taking into account (1.5) and (1.12), we get

$$
\begin{aligned}
& \sum_{i \in \mathbb{Z}^{r}} \theta\left(\frac{\|i\|_{m}}{L}\right)\left(\begin{array}{c}
\frac{|\beta|}{2} \frac{d}{d t} u_{i}^{2}+\frac{|\alpha|}{2} \frac{d}{d t} v_{i}^{2}+|\beta| f_{1}\left(u_{i}\right) u_{i} \\
+|\alpha| f_{2}\left(v_{i}\right) v_{i}+|\beta| g_{1}\left(u_{i}\right) u_{i}+|\alpha| g_{2}\left(v_{i}\right) v_{i}
\end{array}\right) \\
& \quad+|\beta| \nu \sum_{i \in \mathbb{Z}^{r}} \sum_{j=1}^{r}\left(B_{j} u\right)_{i}\left(B_{j} w\right)_{i}=\sum_{i \in \mathbb{Z}^{r}} \theta\left(\frac{\|i\|_{m}}{L}\right)\left(|\beta| q_{1, i} u_{i}+|\alpha| q_{2, i} v_{i}\right) .
\end{aligned}
$$

Using (1.8), (1.10), and the mean value theorem, it follows that for each $i \in \mathbb{Z}^{r}$,

$$
f_{1}\left(u_{i}\right) u_{i} \geq \varepsilon_{1} u_{i}^{2}, \quad f_{2}\left(v_{i}\right) v_{i} \geq \varepsilon_{2} v_{i}^{2}
$$

From (1.11), we obtain

$$
g_{1}\left(u_{i}\right) u_{i} \geq 0, \quad g_{2}\left(v_{i}\right) v_{i} \geq 0
$$

Recalling (69) of [21], taking into account that $\left\|B_{j}\right\| \leq 2, j=1,2, \ldots, r$, one can see that

$$
|\beta| \nu \sum_{i \in \mathbb{Z}^{r}} \sum_{j=1}^{r}\left(B_{j} u\right)_{i}\left(B_{j} w\right)_{i} \geq-\frac{16|\beta| \nu n M_{0}}{L} r_{0}^{2}, \quad \forall t \geq T_{0} .
$$


Now if we substitute (4.6), (4.7), and (4.8), into (4.5), we find that for $t \geq T_{0}$,

$$
\begin{aligned}
\sum_{i \in \mathbb{Z}^{r}} \theta & \left(\frac{\|i\|_{m}}{L}\right)\left(\frac{|\beta|}{2} \frac{d}{d t} u_{i}^{2}+\frac{|\alpha|}{2} \frac{d}{d t} v_{i}^{2}+\varepsilon_{1}|\beta| u_{i}^{2}+\varepsilon_{2}|\alpha| v_{i}^{2}\right) \\
& \leq \sum_{i \in \mathbb{Z}^{r}} \theta\left(\frac{\|i\|_{m}}{L}\right)\left(|\beta| q_{1, i} u_{i}+|\alpha| q_{2, i} v_{i}\right)+\frac{16|\beta| \nu n M_{0}}{L} r_{0}^{2} \\
& \leq \sum_{i \in \mathbb{Z}^{r}} \theta\left(\frac{\|i\|_{m}}{L}\right)\left(\frac{|\beta|}{\varepsilon_{1}} q_{1, i}^{2}+\frac{\varepsilon_{1}|\beta|}{4} u_{i}^{2}+\frac{|\alpha|}{\varepsilon_{2}} q_{2, i}^{2}+\frac{\varepsilon_{2}|\alpha|}{4} v_{i}^{2}\right)+\frac{16|\beta| \nu n M_{0}}{L} r_{0}^{2} .
\end{aligned}
$$

Thus if we choose

$$
\sigma=\min \left\{|\beta|,|\alpha|, \varepsilon_{1}|\beta|, \varepsilon_{2}|\alpha|\right\}
$$

then it follows that for $t \geq T_{0}$,

$$
\begin{aligned}
\sum_{i \in \mathbb{Z}^{r}} \theta & \left(\frac{\|i\|_{m}}{L}\right)\left(\frac{d}{d t}\left\|\varphi_{i}(t)\right\|_{E}^{2}+\left\|\varphi_{i}(t)\right\|_{E}^{2}\right) \\
& \leq 2 \sum_{i \in \mathbb{Z}^{r}} \theta\left(\frac{\|i\|_{m}}{L}\right)\left(\left(\frac{\beta}{\sigma} q_{1, i}\right)^{2}+\left(\frac{\alpha}{\sigma} q_{2, i}\right)^{2}\right)+\frac{32|\beta| \nu n M_{0}}{L} r_{0}^{2} \\
& \leq 2 \sum_{\|i\|_{m} \geq L}\left(\left(\frac{\beta}{\sigma} q_{1, i}\right)^{2}+\left(\frac{\alpha}{\sigma} q_{2, i}\right)^{2}\right)+\frac{32|\beta| \nu n M_{0}}{L} r_{0}^{2} .
\end{aligned}
$$

Since $q_{1}, q_{2} \in l^{2}$, then for a given $\eta>0$, we can fix $L$ such that

$$
2 \sum_{\|i\|_{m} \geq L}\left(\left(\frac{\beta}{\sigma} q_{1, i}\right)^{2}+\left(\frac{\alpha}{\sigma} q_{2, i}\right)^{2}\right)+\frac{32|\beta| \nu n M_{0}}{L} r_{0}^{2} \leq \frac{\eta}{2},
$$

and in such a case we get that

$$
\sum_{i \in \mathbb{Z}^{r}} \theta\left(\frac{\|i\|_{m}}{L}\right)\left(\frac{d}{d t}\left\|\varphi_{i}(t)\right\|_{E}^{2}+\left\|\varphi_{i}(t)\right\|_{E}^{2}\right) \leq \frac{\eta}{2}, \quad \forall t \geq T_{0} .
$$

From the Gronwall lemma, we obtain

$$
\sum_{i \in \mathbb{Z}^{r}}\left(\theta\left(\frac{\|i\|_{m}}{L}\right)\left\|\varphi_{i}(t)\right\|_{E}^{2}\right) \leq e^{-t} \sum_{i \in \mathbb{Z}^{r}}\left(\theta\left(\frac{\|i\|_{m}}{L}\right)\left\|\varphi_{i}(0)\right\|_{E}^{2}\right)+\frac{\eta}{2}, \quad \forall t \geq T_{0} .
$$

Since $\varphi(0)=\left(u_{0}, v_{0}\right)^{T} \in O$, we have

$$
\|\varphi(0)\|_{E} \leq r_{0}
$$

Therefore,

$$
\sum_{i \in \mathbb{Z}^{r}}\left(\theta\left(\frac{\|i\|_{m}}{L}\right)\left\|\varphi_{i}(t)\right\|_{E}^{2}\right) \leq r_{0}^{2} e^{-t}+\frac{\eta}{2}, \quad \forall t \geq T_{0}
$$


But again for $\eta>0$, there exists a constant $T_{1}=T_{1}(\eta)$ such that

$$
r_{0}^{2} e^{-t} \leq \frac{\eta}{2}, \quad \forall t \geq T_{1}
$$

Using (4.16) and (4.17) with $K(\eta)=2 L, T(\eta)=\max \left\{T_{0}, T_{1}\right\}$, we obtain

$$
\begin{aligned}
\sum_{\|i\|_{m} \geq K(\eta)}\left\|\varphi_{i}\right\|_{E}^{2} & =\sum_{\|i\|_{m} \geq K(\eta)}\left(\theta\left(\frac{\|i\|_{m}}{L}\right)\left\|\varphi_{i}\right\|_{E}^{2}\right) \\
& \leq \sum_{i \in \mathbb{Z}^{r}}\left(\theta\left(\frac{\|i\|_{m}}{L}\right)\left\|\varphi_{i}\right\|_{E}^{2}\right) \leq \eta, \quad \forall t \geq T(\eta) .
\end{aligned}
$$

The proof is completed.

Lemma 4.2. If (1.5), (1.7), (1.8), (1.9), (1.10), (1.11), and (1.12) are satisfied, then the solution semigroup $\{S(t)\}_{t \geq 0}$ of (2.9) is asymptotically compact in $E$, that is, if $\left\{\varphi_{n}\right\}$ is bounded in $E$ and $t_{n} \rightarrow \infty$, then $\left\{S\left(t_{n}\right) \varphi_{n}\right\}$ is precompact in $E$.

Proof. By using Lemmas 3.2 and 4.1, above, the proof of this lemma will be similar to that of [19, Lemma 3.2].

Theorem 4.3. If (1.5), (1.7), (1.8), (1.9), (1.10), (1.11), and (1.12) are satisfied, then the solution semigroup $\{S(t)\}_{t \geq 0}$ of (2.9) possesses a global attractor $\mathcal{A}$ in $E$.

Proof. From the existence theorem of global attractors, (cf. [18, Lemmas 2 and 4]), we get the result.

\section{Upper semicontinuity of the global attractor}

Here we will study the upper semicontinuity of the global attractor $\mathscr{A}$ of the solution semigroup $\{S(t)\}_{t \geq 0}$ of (2.9), in the sense that $\mathscr{A}$ is approximated by the global attractors of finite-dimensional versions of (2.9), as was done in [4] for a simpler system.

Let $n$ be a positive integer, and

$$
\mathbb{Z}_{n}^{r}=\left\{i \in \mathbb{Z}^{r}:\|i\|_{m} \leq n\right\}
$$

For $i=\left(i_{1}, i_{2}, \ldots, i_{r}\right) \in \mathbb{Z}_{n}^{r}$, consider $w=\left(w_{i}\right)_{\|i\|_{m} \leq n} \in \mathbb{R}^{(2 n+1)^{r}}$. For convenience, we reorder the subscripts of components of $w$ as follows:

$$
\begin{aligned}
w= & \left(w_{(-n,-n, \ldots,-n,-n)}, w_{(-n,-n, \ldots,-n,-n+1)}, \ldots, w_{(-n,-n, \ldots,-n, n)},\right. \\
& w_{(-n,-n, \ldots,-n+1,-n)}, w_{(-n,-n, \ldots,-n+1,-n+1)}, \ldots, \\
& \left.w_{(-n,-n, \ldots,-n+1, n)}, \ldots, w_{(n, n, \ldots, n,-n)}, w_{(n, n, \ldots, n,-n+1)}, \ldots, w_{(n, n, \ldots, n, n)}\right)^{T} .
\end{aligned}
$$

Let

$$
X=w=\left(w_{i}\right)_{\|i\|_{m} \leq n}: w \in \mathbb{R}^{(2 n+1)^{r}},
$$

where subscripts of components of $w$ are ordered as in (5.2). 
Consider the $(2 n+1)^{r}$-dimensional ordinary differential equations with the initial data in $X$ :

$$
\begin{gathered}
\dot{w}_{i}+v(A w)_{i}+f_{1}\left(w_{i}\right)+g_{1}\left(w_{i}\right)+\alpha h_{1}\left(w_{i}, z_{i}\right)=q_{1, i} \\
\dot{z}_{i}+f_{2}\left(z_{i}\right)+g_{2}\left(z_{i}\right)-\beta h_{2}\left(w_{i}, z_{i}\right)=q_{2, i}, \\
w_{i}(0)=w_{i, 0}, \quad z_{i}(0)=z_{i, 0} \\
i \in \mathbb{Z}_{n}^{r}, t>0 .
\end{gathered}
$$

The system (5.4) can be written as

$$
\begin{gathered}
\dot{w}+v \tilde{A} w+\tilde{f}_{1}(w)+\tilde{g}_{1}(w)+\alpha \tilde{h}_{1}(w, z)=\tilde{q}_{1}, \\
\dot{z}+\tilde{f}_{2}(z)+\tilde{g}_{2}(z)-\beta \tilde{h}_{2}(w, z)=\tilde{q}_{2}, \\
w(0)=\left(w_{i, 0}\right)_{\|i\|_{m} \leq n}, \quad z(0)=\left(z_{i, 0}\right)_{\|i\|_{m} \leq n},
\end{gathered}
$$

where $w=\left(w_{i}\right)_{\|i\|_{m} \leq n}, z=\left(z_{i}\right)_{\|i\|_{m} \leq n} \in X$, for $k=1,2, \ldots, r$,

$$
\begin{gathered}
w_{\left(i_{1}, \ldots,-n, i_{k}+1, \ldots, i_{r}\right)}=w_{\left(i_{1}, \ldots, n+1, i_{k}+1, \ldots, i_{r}\right)}, \\
w_{\left(i_{1}, \ldots, n, i_{k}+1, \ldots, i_{r}\right)}=w_{\left(i_{1}, \ldots,-n-1, i_{k}+1, \ldots, i_{r}\right)}, \\
(\tilde{A} w)_{\left(i_{1}, i_{2}, \ldots, i_{r}\right)}=2 r w_{\left(i_{1}, i_{2}, \ldots, i_{r}\right)}-w_{\left(i_{1}-1, i_{2}, \ldots, i_{r}\right)}-w_{\left(i_{1}, i_{2}-1, \ldots, i_{r}\right)}-\cdots-w_{\left(i_{1}, i_{2}, \ldots, i_{r}-1\right)} \\
-w_{\left(i_{1}+1, i_{2}, \ldots, i_{r}\right)}-w_{\left(i_{1}, i_{2}+1, \ldots, i_{r}\right)}-\cdots-w_{\left(i_{1}, i_{2}, \ldots, i_{r}+1\right)},
\end{gathered}
$$

and for $j=1,2$,

$$
\begin{aligned}
\tilde{f}_{j}(w) & =\left(f_{j}\left(w_{i}\right)\right)_{\|i\|_{m} \leq n}, & \tilde{g}_{j}(w) & =\left(g_{j}\left(w_{i}\right)\right)_{\|i\|_{m} \leq n}, \\
\widetilde{h_{j}}(w, z) & =\left(h_{j}\left(w_{i}, z_{i}\right)\right)_{\|i\|_{m} \leq n}, & \tilde{q}_{j} & =\left(q_{j, i}\right)_{\|i\|_{m} \leq n} .
\end{aligned}
$$

For $w=\left(w_{i}\right)_{\|i\|_{m} \leq n} \in X$, define the linear operators $\widetilde{B}_{j}, \widetilde{B_{j}^{*}}, \widetilde{A}_{j}: X \rightarrow X, j=1,2, \ldots, r$, by

$$
\begin{aligned}
& \left(\widetilde{B}_{j} w\right)_{i}=w_{\left(i_{1}, \ldots, i_{j}+1, \ldots, i_{r}\right)}-w_{\left(i_{1}, \ldots, i_{j}, \ldots, i_{r}\right)}, \\
& \left(\widetilde{B_{j}^{*}} w\right)_{i}=w_{\left(i_{1}, \ldots, i_{j}-1, \ldots, i_{r}\right)}-w_{\left(i_{1}, \ldots, i_{j}, \ldots, i_{r}\right)}, \\
& \left(\widetilde{A}_{j} w\right)_{i}=2 w_{\left(i_{1}, \ldots, i_{j}, \ldots, i_{r}\right)}-w_{\left(i_{1}, \ldots, i_{j}-1, \ldots, i_{r}\right)}-w_{\left(i_{1}, \ldots, i_{j}+1, \ldots, i_{r}\right)},
\end{aligned}
$$

then

$$
\widetilde{A}=\widetilde{A}_{1}+\widetilde{A}_{2}+\cdots+\widetilde{A}_{r}, \quad \widetilde{A}_{j}=\widetilde{B}_{j} \widetilde{B}_{j}^{*}=\widetilde{B_{j}^{*}} \widetilde{B}_{j}, \quad j=1,2, \ldots, r
$$

For $w^{(j)}=\left(w_{i}^{(j)}\right)_{\|i\|_{m} \leq n} \in X, j=1,2, i=\left(i_{1}, i_{2}, \ldots, i_{r}\right) \in \mathbb{Z}_{n}^{r}$, define

$$
\left\langle w^{(1)}, w^{(2)}\right\rangle_{X}=\sum_{\|i\|_{m} \leq n} w_{i}^{(1)} w_{i}^{(2)}, \quad\left\|w^{(1)}\right\|_{X}=\left\langle w^{(1)}, w^{(1)}\right\rangle_{X}^{1 / 2}
$$


In such a case, it is clear that $X=\left(X,\|\cdot\|_{X}\right)$ is a Hilbert space, and $\widetilde{E}=X \times X$ is a Hilbert space with the following inner product and norm: for $Y_{j}=\left(w^{(j)}, z^{(j)}\right)^{T}=\left(\left(w_{i}^{(j)}\right)\right.$, $\left.\left(z_{i}^{(j)}\right)\right)_{\|i\|_{m} \leq n}^{T} \in \widetilde{E}, j=1,2$,

$$
\left\langle Y_{1}, Y_{2}\right\rangle_{\widetilde{E}}=\left\langle w^{(1)}, w^{(2)}\right\rangle_{X}+\left\langle z^{(1)}, z^{(2)}\right\rangle_{X}, \quad\left\|Y_{1}\right\|_{\widetilde{E}}=\left(\left\|w^{(1)}\right\|_{X}^{2}+\left\|z^{(1)}\right\|_{X}^{2}\right)^{1 / 2} .
$$

It is easy to check that problem (5.5) can be formulated to the following first-order system in the Hilbert space $\widetilde{E}$ :

$$
\dot{Y}+\widetilde{C}(Y)=\widetilde{F}(Y), \quad Y(0)=(w(0), z(0))^{T} \in \widetilde{E},
$$

where

$$
\begin{gathered}
Y=(w, z)^{T}, \quad \tilde{C}(Y)=(\nu \tilde{A} w, 0)^{T}, \quad \tilde{F}(Y)=\left(\widetilde{G}_{1}(Y), \widetilde{G}_{2}(Y)\right)^{T}, \\
\tilde{G}_{1}(Y)=\tilde{q}_{1}-\lambda \tilde{f}_{1}(w)-\tilde{g}_{1}(w)-\alpha \tilde{h}_{1}(w, z), \\
\tilde{G}_{2}(Y)=\tilde{q}_{2}-\delta \tilde{f}_{2}(z)-\tilde{g}_{2}(z)+\beta \tilde{h}_{2}(w, z) .
\end{gathered}
$$

Similar to Section 2, one can see that, if (1.5), (1.7), (1.8), (1.9), (1.10), (1.11), and (1.12) are satisfied, then $(5.12)$ is a well-posed problem in $\widetilde{E}$. Thus for any $Y(0) \in \widetilde{E}$, there exists a unique solution $Y \in C([0,+\infty), \widetilde{E}) \cap C^{1}((0,+\infty), \widetilde{E})$, see Lemmas 3.1 and 3.2 , also there exist maps of solutions $S_{n}(t): Y(0) \in \widetilde{E} \rightarrow Y(t)=S_{n}(t) Y(0) \in \widetilde{E}, t \geq 0$, generating a continuous semigroup $\left\{S_{n}(t)\right\}_{t \geq 0}$ on $\widetilde{E}$.

Similar to Lemma 3.2 and Theorem 4.3, we can prove the following Lemma.

Lemma 5.1. If (1.5), (1.7), (1.8), (1.9), (1.10), (1.11), and (1.12) are satisfied, then there exists a bounded ball $\widetilde{O}=\widetilde{O}_{\widetilde{E}}\left(0, r_{0}\right)$ in $\widetilde{E}$, centered at 0 with radius $r_{0}$ such that for every bounded set $\tilde{G}$ of $\widetilde{E}$, there exists $T(\widetilde{G}) \geq 0$ such that

$$
S_{n}(t) \widetilde{G} \subset \widetilde{O}, \quad \forall t \geq T(\widetilde{G}), n=1,2, \ldots,
$$

where $r_{0}$ is the same constant given by Lemma 3.2, and it is independent of $n$. Moreover, the semigroup $\left\{S_{n}(t)\right\}_{t \geq 0}$ possesses a global attractor $\mathscr{A}_{n}, \mathscr{A}_{n} \subset \widetilde{O} \subset \widetilde{E}$.

Here we prove the upper semicontinuity of the global attractor $\mathscr{A}$ of the solution semigroup $\{S(t)\}_{t \geq 0}$ of (2.9). In such a case, we should extend the element $u=\left(u_{i}\right)_{\|i\|_{m} \leq n} \in X$ to an element of $l^{2}$ such that $u_{i}=0$ for $\|i\|_{m}>n$, still denoted by $u$.

Lemma 5.2. If (1.5), (1.7), (1.8), (1.9), (1.10), (1.11), and (1.12) are satisfied, and $\varphi_{n}(0) \in$ $\mathscr{A}_{n}$, then there exists a subsequence $\left\{\varphi_{n_{k}}(0)\right\}$ of $\left\{\varphi_{n}(0)\right\}$ and $\varphi_{0} \in \mathcal{A}$ such that $\varphi_{n_{k}}(0)$ converges to $\varphi_{0}$ in $E$.

Proof. Consider $\varphi_{n}(t)=S_{n}(t) \varphi_{n}(0)=\left(u_{n}(t), v_{n}(t)\right)^{T} \in \widetilde{E}$ to be a solution of (5.12). Since $\varphi_{n}(0) \in \mathscr{A}_{n}, \varphi_{n}(t) \in \mathscr{A}_{n} \subset \widetilde{O}$ for all $t \in \mathbb{R}^{+}$, and again the element $\varphi_{n}=\left(\varphi_{n, i}\right)_{\|i\|_{m} \leq n} \in \widetilde{E}$ can be extended to the element $\varphi_{n}=\left(\varphi_{n, i}\right)_{i \in \mathbb{Z}^{r}} \in E$, where $\varphi_{n, i}=(0,0)^{T}$ for $\|i\|_{m}>n$, it 
follows that

$$
\begin{aligned}
\left\|\varphi_{n}(t)\right\|_{\tilde{E}} & =\left\|\varphi_{n}(t)\right\|_{E}=\left(\left\|u_{n}\right\|^{2}+\left\|v_{n}\right\|^{2}\right)^{1 / 2} \\
& \leq r_{0}, \quad \forall t \in \mathbb{R}^{+}, n=1,2, \ldots
\end{aligned}
$$

From (2.5) and (5.15), we get that

$$
\left\|\widetilde{C}\left(\varphi_{n}(t)\right)\right\|_{\widetilde{E}}^{2} \leq\left\|C\left(\varphi_{n}(t)\right)\right\|_{E}^{2}=\left\|\nu A u_{n}\right\|^{2} \leq \nu C_{0}\left\|u_{n}\right\|^{2} \leq \nu C_{0} r_{0}^{2}
$$

for all $t \in \mathbb{R}^{+}, n=1,2, \ldots$.

Similarly, for $j=1,2$, the element $\tilde{q}_{j}=\left(q_{j, i}\right)_{\|i\|_{m} \leq n} \in \mathbb{R}^{(2 n+1)^{r}}$ can be extended to the element $q_{n, j}=\left(q_{n, j, i}\right)_{i \in \mathbb{Z}^{r}} \in l^{2}$, where $q_{n, j, i}=q_{j, i}$ for $\|i\|_{m} \leq n$ and $q_{n, j, i}=0$ for $\|i\|_{m}>n$. From (1.8) and (1.9), we know that for $j=1,2$,

$$
f_{j}(0)=g_{j}(0)=h_{j}(0,0)=0 \text {. }
$$

Therefore,

$$
\begin{aligned}
\left\|\widetilde{F}\left(\varphi_{n}(t)\right)\right\|_{\widetilde{E}}^{2}= & \left\|F\left(\varphi_{n}(t)\right)\right\|_{E}^{2}=\left\|q_{n, 1}-f_{1}\left(u_{n}\right)-g_{1}\left(u_{n}\right)-\alpha h_{1}\left(u_{n}, v_{n}\right)\right\|^{2} \\
& +\left\|q_{n, 2}-f_{2}\left(v_{n}\right)-g_{2}\left(v_{n}\right)+\beta h_{2}\left(u_{n}, v_{n}\right)\right\|^{2} \\
\leq & 2\left\|q_{1}\right\|^{2}+4\left\|f_{1}\left(u_{n}\right)\right\|^{2}+8\left\|g_{1}\left(u_{n}\right)\right\|^{2}+8 \alpha^{2}\left\|h_{1}\left(u_{n}, v_{n}\right)\right\|^{2} \\
& +2\left\|q_{2}\right\|^{2}+4\left\|f_{2}\left(v_{n}\right)\right\|^{2}+8\left\|g_{2}\left(v_{n}\right)\right\|^{2}+8 \beta^{2}\left\|h_{2}\left(u_{n}, v_{n}\right)\right\|^{2} .
\end{aligned}
$$

Using (1.9), (5.15), and the mean value theorem, there exist $\xi_{6 i}, \xi_{7 i} \in(0,1)$ for each $i \in \mathbb{Z}^{r}$ such that

$$
\begin{aligned}
\left\|h_{1}\left(u_{n}, v_{n}\right)\right\|^{2} & =\sum_{i \in \mathbb{Z}^{r}}\left(h_{1}\left(u_{n, i}, v_{n, i}\right)\right)^{2} \\
& =\sum_{i \in \mathbb{Z}^{r}}\left(D_{1} h_{1}\left(\xi_{6 i} u_{n, i}, v_{n, i}\right) u_{n, i}+D_{2} h_{1}\left(0, \xi_{7 i} v_{n, i}\right) v_{n, i}\right)^{2} \\
& \leq 2\left(\max _{a, b \in\left[-r_{0}, r_{0}\right]}\left(D_{1} h_{1}(a, b)\right)^{2}\right) r_{0}^{2}+2\left(\max _{b \in\left[-r_{0}, r_{0}\right]}\left(D_{2} h_{1}(0, b)\right)^{2}\right) r_{0}^{2}
\end{aligned}
$$

for all $t \in \mathbb{R}^{+}, n=1,2, \ldots$ Again using (1.8), (1.9), (5.15), and the mean value theorem, we can get similar results, as (5.19), for $f_{1}, f_{2}, g_{1}, g_{2}$, and $h_{2}$. Thus from (5.18), it is obvious that there exists a constant $C_{1}=C_{1}\left(r_{0}, q_{1}, q_{2}, f_{1}, f_{2}, g_{1}, g_{2}, h_{1}, h_{2}\right)$ such that

$$
\left\|\widetilde{F}\left(\varphi_{n}(t)\right)\right\|_{\widetilde{E}}^{2}=\left\|F\left(\varphi_{n}(t)\right)\right\|_{E}^{2} \leq C_{1}, \quad \forall t \in \mathbb{R}^{+}, n=1,2, \ldots
$$

Now, by using (5.12), we obtain

$$
\left\|\dot{\varphi}_{n}(t)\right\|_{E}^{2} \leq 2\left\|C\left(\varphi_{n}(t)\right)\right\|_{E}^{2}+2\left\|F\left(\varphi_{n}(t)\right)\right\|_{E}^{2}
$$

Hence, from (5.16) and (5.20), it follows that there exists a constant $C_{2}=C_{2}\left(r_{0}, C_{0}, C_{1}\right)$ such that

$$
\left\|\dot{\varphi}_{n}(t)\right\|_{E} \leq C_{2}, \quad \forall t \in \mathbb{R}^{+}, n=1,2, \ldots
$$


Let $J_{k}(k=1,2, \ldots)$ be a sequence of compact intervals of $\mathbb{R}^{+}$such that $J_{k} \subset J_{k+1}$ and $\cup_{k} J_{k}=\mathbb{R}^{+}$. Then there exists a subsequence of $\left\{\varphi_{n}(t)\right\}$, still denoted by $\left\{\varphi_{n}(t)\right\}$, and $\varphi(t) \in C\left(\mathbb{R}^{+}, E\right)$ such that

$$
\begin{gathered}
\varphi_{n}(t) \longrightarrow \varphi(t) \text { in } C(J, E) \text { as } n \longrightarrow \infty \text { for any compact set } J \subset \mathbb{R}^{+}, \\
\dot{\varphi}_{n}(t) \longrightarrow \dot{\varphi}(t) \text { weak star in } L^{\infty}\left(\mathbb{R}^{+}, E\right) \text { as } n \longrightarrow+\infty
\end{gathered}
$$

From (5.15) and (5.23), we obtain that there exists a constant $C_{3}=C_{3}\left(r_{0}\right)$ such that for $\varphi(t)=(u(t), v(t))^{T}=\left(\left(u_{i}(t)\right),\left(v_{i}(t)\right)\right)_{i \in \mathbb{Z}^{r}}^{T} \in E$,

$$
\|\varphi(t)\|_{E}=\left(\|u\|^{2}+\|v\|^{2}\right)^{1 / 2} \leq C_{3}, \quad \forall t \in \mathbb{R}^{+} .
$$

Let $i \in \mathbb{Z}^{r}$ and $n \geq\|i\|_{m}$. Since $\varphi_{n}(t)=\left(u_{n}(t), v_{n}(t)\right)^{T}=\left(\left(u_{n, i}(t)\right),\left(v_{n, i}(t)\right)\right)_{\|i\|_{m} \leq n}^{T} \in \widetilde{E}$ is the solution of (5.12), it follows that for all $t \in \mathbb{R}^{+}$and $i \in \mathbb{Z}_{n-1}^{r}$,

$$
\begin{gathered}
\dot{u}_{n, i}+v\left(A u_{n}\right)_{i}+f_{1}\left(u_{n, i}\right)+g_{1}\left(u_{n, i}\right)+\alpha h_{1}\left(u_{n, i}, v_{n, i}\right)=q_{1, i}, \\
\dot{v}_{n, i}+f_{2}\left(v_{n, i}\right)+g_{2}\left(v_{n, i}\right)-\beta h_{2}\left(u_{n, i}, v_{n, i}\right)=q_{2, i} .
\end{gathered}
$$

Therefore, for all $\psi \in C_{0}^{\infty}(J)$, we obtain

$$
\begin{gathered}
\int_{J}\left(\dot{u}_{n, i}+\nu\left(A u_{n}\right)_{i}+f_{1}\left(u_{n, i}\right)+g_{1}\left(u_{n, i}\right)+\alpha h_{1}\left(u_{n, i}, v_{n, i}\right)\right) \psi(t) d t=\int_{J} q_{1, i} \psi(t) d t \\
\int_{J}\left(\dot{v}_{n, i}+f_{2}\left(v_{n, i}\right)+g_{2}\left(v_{n, i}\right)-\beta h_{2}\left(u_{n, i}, v_{n, i}\right)\right) \psi(t) d t=\int_{J} q_{2, i} \psi(t) d t .
\end{gathered}
$$

From (1.8), and by using the mean value theorem, there exists $\xi_{8 i} \in(0,1)$ for each $i$ such that

$$
\begin{aligned}
& \left|\int_{J} f_{1}\left(u_{n, i}\right) \psi(t) d t-\int_{J} f_{1}\left(u_{i}\right) \psi(t) d t\right| \\
& \quad \leq \sup _{t \in J}\left|f_{1}\left(u_{n, i}\right)-f_{1}\left(u_{i}\right)\right| \int_{J}|\psi(t)| d t \\
& \quad \leq \sup _{t \in J}\left(\left|f_{1}^{\prime}\left(u_{n, i}+\xi_{8 i}\left(u_{i}-u_{n, i}\right)\right)\right|\left|u_{n, i}-u_{i}\right|\right) \int_{J}|\psi(t)| d t .
\end{aligned}
$$

By using (5.15) and (5.25), it is clear that there exists a constant $C_{4}=C_{4}\left(r_{0}\right)$ such that

$$
\left|u_{n, i}+\xi_{i}\left(u_{i}-u_{n, i}\right)\right| \leq\left|u_{n, i}\right|+\left|u_{i}\right| \leq\left\|u_{n}||+\right\| u \| \leq C_{4}
$$

for all $t \in \mathbb{R}^{+}$and $n=1,2, \ldots$ In such a case, we obtain

$$
\sup _{t \in J}\left|f_{1}^{\prime}\left(u_{n, i}+\xi_{8 i}\left(u_{i}-u_{n, i}\right)\right)\right| \leq \sup _{a \in\left[-C_{5}, C_{5}\right]}\left|f_{1}^{\prime}(a)\right|<+\infty .
$$

From (5.23), we know that as $n \rightarrow \infty$, we have

$$
\sup _{t \in J}\left|u_{n, i}-u_{i}\right| \longrightarrow 0
$$


Therefore, from (5.28), (5.30), and (5.31), it is clear that

$$
\left|\int_{J} f_{1}\left(u_{n, i}\right) \psi(t) d t-\int_{J} f_{1}\left(u_{i}\right) \psi(t) d t\right| \longrightarrow 0, \quad \text { as } n \longrightarrow \infty .
$$

Similarly, one can get the same result, as (5.32), for $f_{2}, g_{1}, g_{2}, h_{1}$, and $h_{2}$. In such a case from (5.24), (5.26), and (5.27), it follows that

$$
\begin{gathered}
\dot{u}_{i}+v(A u)_{i}+\lambda f_{1}\left(u_{i}\right)+g_{1}\left(u_{i}\right)+\alpha h_{1}\left(u_{i}, v_{i}\right)=q_{1, i}, \\
\dot{v}_{i}+\delta f_{2}\left(v_{i}\right)+g_{2}\left(v_{i}\right)-\beta h_{2}\left(u_{i}, v_{i}\right)=q_{2, i} .
\end{gathered}
$$

But $J$ is arbitrary, thus (5.33) holds for all $t \in \mathbb{R}^{+}$, which means that $\varphi(t)$ is a solution of (2.9). From (5.25), it follows that $\varphi(t)$ is bounded for $t \in \mathbb{R}^{+}$, that is, $\varphi(t) \in \mathscr{A}$, therefore $\varphi_{n}(0) \rightarrow \varphi(0) \in \mathscr{A}$, and the proof is completed.

Now we are ready to represent the main result of this section. In fact, the following theorem shows that the global attractor $\mathscr{A}$ of the lattice dynamical system (2.9) is upper semicontinuous with respect to the (cutoff) approximate finite-dimensional dynamical system (5.12).

Theorem 5.3. If (1.5), (1.7), (1.8), (1.9), (1.10), (1.11), and (1.12) are satisfied, then

$$
\lim _{n \rightarrow \infty} d_{E}\left(\mathscr{A}_{n}, \mathscr{A}\right)=0
$$

where $d_{E}\left(\mathscr{A}_{n}, \mathscr{A}\right)=\sup _{a \in \mathscr{A}_{n}} \inf _{b \in \mathscr{A}}\|a-b\|_{E}$.

Proof. We argue by contradiction. If the conclusion is not true, then there exists a sequence $\varphi_{n_{k}} \in \mathscr{A}_{n_{k}}$ and a constant $K>0$ such that

$$
d_{E}\left(\varphi_{n_{k}}, \mathscr{A}\right) \geq K>0
$$

However, by Lemma 5.2, we know that there exists a subsequence $\varphi_{n_{k_{m}}}$ of $\varphi_{n_{k}}$ such that

$$
d_{E}\left(\varphi_{n_{k m}}, \mathscr{A}\right) \longrightarrow 0
$$

which contradicts (5.35). The proof is completed.

\section{References}

[1] V. S. Afraimovich, S.-N. Chow, and J. K. Hale, Synchronization in lattices of coupled oscillators, Phys. D 103 (1997), no. 1-4, 442-451.

[2] V. S. Afraimovich and V. I. Nekorkin, Chaos of traveling waves in a discrete chain of diffusively coupled maps, Internat. J. Bifur. Chaos Appl. Sci. Engrg. 4 (1994), no. 3, 631-637.

[3] P. W. Bates and A. Chmaj, A discrete convolution model for phase transitions, Arch. Ration. Mech. Anal. 150 (1999), no. 4, 281-305.

[4] P. W. Bates, K. Lu, and B. Wang, Attractors for lattice dynamical systems, Internat. J. Bifur. Chaos Appl. Sci. Engrg. 11 (2001), no. 1, 143-153.

[5] J. Bell, Some threshold results for models of myelinated nerves, Math. Biosci. 54 (1981), no. 3-4, 181-190. 
[6] J. Bell and C. Cosner, Threshold behavior and propagation for nonlinear differential-difference systems motivated by modeling myelinated axons, Quart. Appl. Math. 42 (1984), no. 1, 114.

[7] S.-N. Chow and J. Mallet-Paret, Pattern formation and spatial chaos in lattice dynamical systems. I, IEEE Trans. Circuits Systems I Fund. Theory Appl. 42 (1995), no. 10, 746-751.

[8] , Pattern formation and spatial chaos in lattice dynamical systems. II, IEEE Trans. Circuits Systems I Fund. Theory Appl. 42 (1995), no. 10, 752-756.

[9] S.-N. Chow, J. Mallet-Paret, and W. Shen, Traveling waves in lattice dynamical systems, J. Differential Equations 149 (1998), no. 2, 248-291.

[10] S.-N. Chow, J. Mallet-Paret, and E. S. Van Vleck, Pattern formation and spatial chaos in spatially discrete evolution equations, Random Comput. Dynam. 4 (1996), no. 2-3, 109-178.

[11] R. Dogaru and L. O. Chua, Edge of chaos and local activity domain of FitzHugh-Nagumo equation, Internat. J. Bifur. Chaos Appl. Sci. Engrg. 8 (1998), no. 2, 211-257.

[12] R. FitzHugh, Impulses and physiological states in theoretical models of nerve membrane, Biophys. J. 1 (1961), 445-466.

[13] J. P. Keener, Propagation and its failure in coupled systems of discrete excitable cells, SIAM J. Appl. Math. 47 (1987), no. 3, 556-572.

[14] M. Marion, Finite-dimensional attractors associated with partly dissipative reaction-diffusion systems, SIAM J. Math. Anal. 20 (1989), no. 4, 816-844.

[15] J. Nagumo, S. Arimoto, and S. Yosimzawa, An active pulse transmission line simulating nerve axon, Proc. I. R. E. 50 (1964), 2061-2070.

[16] A. Rodriguez-Bernal and B. Wang, Attractors for partly dissipative reaction diffusion systems in $\mathbf{R}^{n}$, J. Math. Anal. Appl. 252 (2000), no. 2, 790-803.

[17] W. Shen, Lifted lattices, hyperbolic structures, and topological disorders in coupled map lattices, SIAM J. Appl. Math. 56 (1996), no. 5, 1379-1399.

[18] R. Temam, Infinite-Dimensional Dynamical Systems in Mechanics and Physics, Applied Mathematical Sciences, vol. 68, Springer-Verlag, New York, 1988.

[19] S. Zhou, Attractors for second order lattice dynamical systems, J. Differential Equations 179 (2002), no. 2, 605-624.

[20] _ Attractors for first order dissipative lattice dynamical systems, Phys. D 178 (2003), no. 12, 51-61.

[21] Attractors and approximations for lattice dynamical systems, J. Differential Equations 200 (2004), no. 2, 342-368.

[22] B. Zinner, Existence of traveling wavefront solutions for the discrete Nagumo equation, J. Differential Equations 96 (1992), no. 1, 1-27.

Ahmed Y. Abdallah: Department of Mathematics, University of Jordan, Amman 11942, Jordan E-mail address: farah@ju.edu.jo 


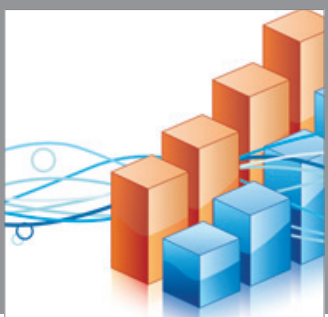

Advances in

Operations Research

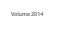

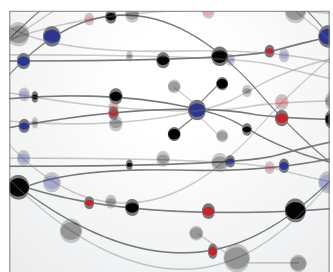

\section{The Scientific} World Journal
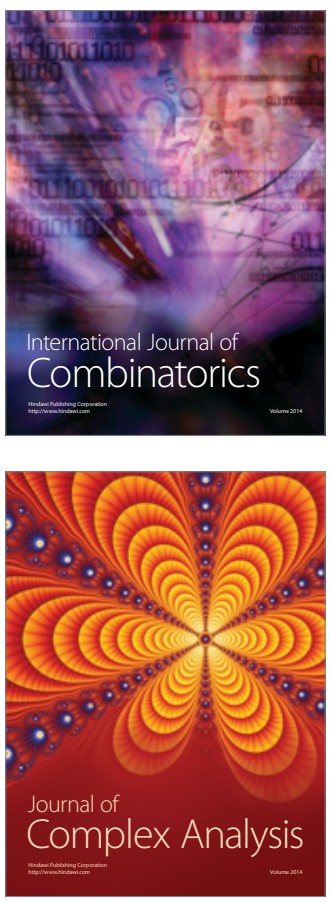

International Journal of

Mathematics and

Mathematical

Sciences
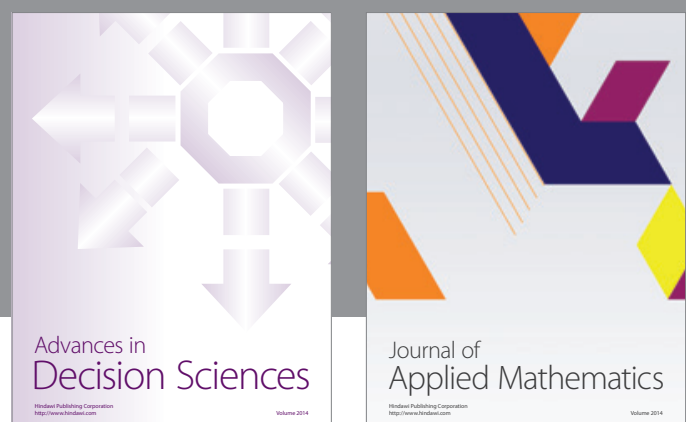

Journal of

Applied Mathematics
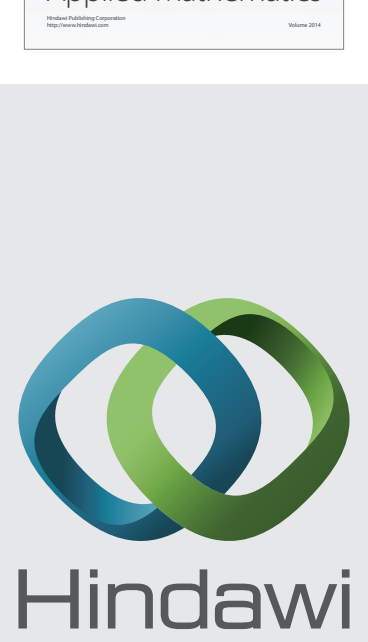

Submit your manuscripts at http://www.hindawi.com
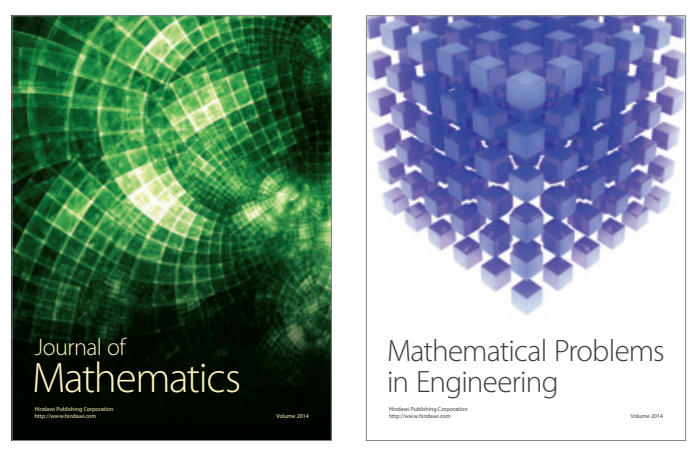

Mathematical Problems in Engineering
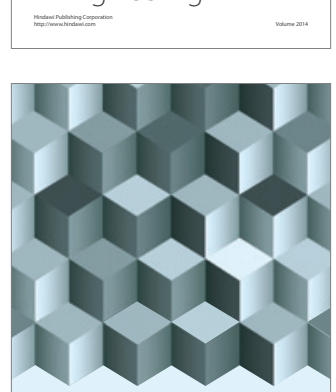

Journal of

Function Spaces
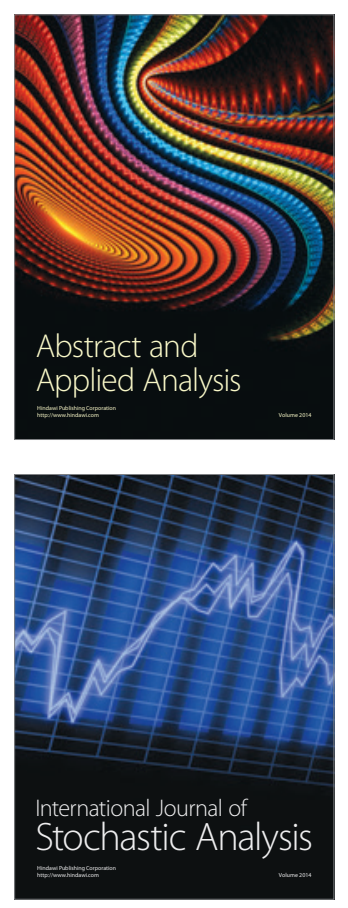

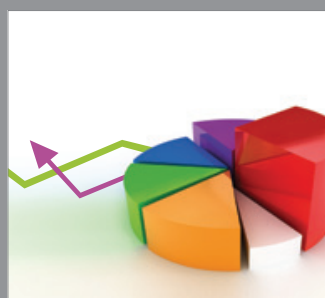

ournal of

Probability and Statistics

Promensencen
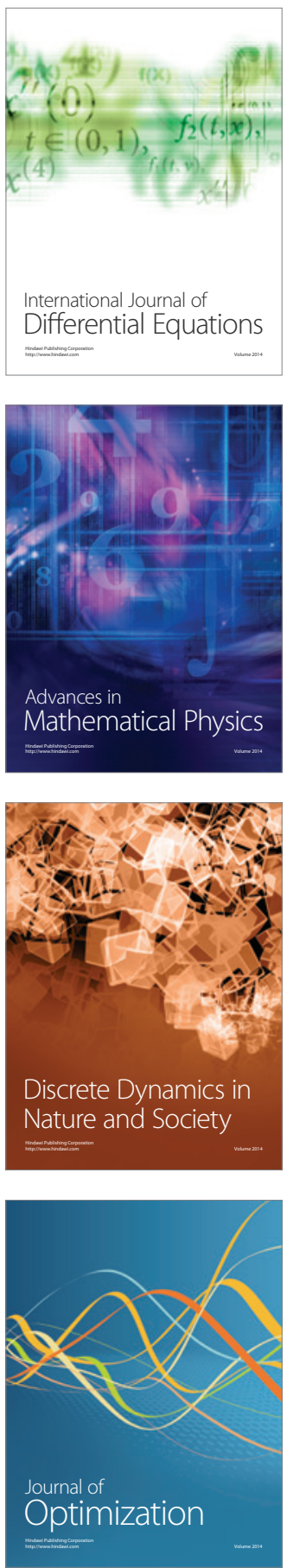